\title{
Coordinated Analysis of a Metal-rich Nugget from a Calcium-aluminum-rich Inclusion.
}

Tarunika Ramprasad ${ }^{1}$, Venkateshwara Manga ${ }^{2}$, Laura Seifert ${ }^{3}$ and Thomas Zega ${ }^{4}$

${ }^{1}$ Materials Science and Engineering, University of Arizona, Tucson, Arizona, United States, ${ }^{2}$ Materials Sceince and Engineering, University of Arizona, United States, ${ }^{3}$ Lunar and Planetary Laboratory, University of Arizona, Tucson, Arizona, United States, ${ }^{4}$ Lunar and Planetary Laboratory, University of Arizona, United States

Calcium-aluminum-rich inclusions (CAIs) are $\mathrm{mm}$ - to $\mathrm{cm}$-sized, ceramic-like objects found in chondritic meteorites, composed of $\mathrm{Ca}$ - and Al-rich mineral phases [1]. The components of CAIs are predicted by equilibrium thermodynamics to condense at high-temperatures from a gas of solar composition [2-3]. Isotopic analyses place the age of CAI minerals at 4.567 Gya and so they are recognized as the first-formed solids in the solar protoplanetary disk [4-5]. Imprinted on their micro to atomic-scale structures are records of the earliest processes in our solar system. Detailed analysis of CAIs can therefore provide information on their origins and chemical and physical processes that occurred in the early solar system.

Inclusions rich in high-Z elements are observed in some CAIs [1]. Refractory metal nuggets (RMNs) are submicron- to micron-sized, Fe- and Ni-rich grains that contain other refractory siderophile elements such as $\mathrm{Pt}$, Ir, Os, Re and Mo [1]. Early work on these objects suggested that they are single-phase alloys, rich in Pt, Os, Ir and $\mathrm{Ru}$ [6-7]. Such refractory metals are predicted to condense at very high temperatures (e.g., Os $\mathrm{T}_{\mathrm{c}}=1917$ $\mathrm{K}[8])$, exceeding even those temperatures at which other major CAI phase such as hibonite $\left(\mathrm{CaAl}_{12} \mathrm{O}_{19}, \mathrm{~T}_{\mathrm{c}}=\right.$ $1659 \mathrm{~K}$ [2]) are predicted to form [3, 8-10]. RMNs can therefore serve as unique thermal probes of some of the highest temperature processes in the solar protoplanetary disk. Here we report a coordinated analytical study, combining several electron- and ion-beam methods, to characterize the structure and composition of these RMNs.

A CAI (Fig. 1a) was identified in a thin section of the Northwest Africa (NWA) 8323 CV3 chondrite (Center for Meteorite Studies, Arizona State University collection, \#1895_1_2) using the Cameca SX-100 electron microprobe (EMP), located in the Kuiper Materials Imaging and Characterization Facility (KMICF) at the Lunar and Planetary Laboratory, University of Arizona. Wavelength-dispersive spectroscopy (WDS) was used to identify grains rich in transition and rare-earth metals. One of these grains 'Bright2' (Fig. 1b) was selected for analysis in the TEM and extracted and thinned to electron transparency following previously described methods [11], using the Thermo Scientific (formerly FEI) Helios NanoLab 660 G3 focused-ion beam (FIB) scanning-electron microscope (SEM) located in the KMICF. The section was analyzed using the $200 \mathrm{keV}$ spherical-aberration corrected Hitachi HF5000 scanning transmission electron microscope (S/TEM) located in the KMICF. The HF5000 is equipped with twin Oxford Instruments (X-max) EDS detectors, providing a 2.0 sr solid angle for X-ray collection. Selected-area electron diffraction (SAED) was used for determination of crystallinity and phase identification.

Microprobe mapping and quantitative analysis shows that the identified CAI ('Cloud') is a fluffy type A (FTA, Fig. 1a). Bright 2 is an elliptical inclusion that occurs within the melilite interior of Cloud (Fig. 1b) and measures approximately $1.1 \mu \mathrm{m} \times 900 \mathrm{~nm}$ in size. Its small size precludes a single-phase quantitative WDS measurement due to the X-ray excitation volume $(\approx 3 \mu \mathrm{m})$ but EDS spot analysis on the EMP showed the presence of $\mathrm{Fe}, \mathrm{Ni}, \mathrm{Pt}$, Ir and Os. We extracted an electron transparent section of Bright2, transecting its longest dimension, to determine its chemical composition and crystal structure with TEM and gain insight into its high-temperature origin.

STEM high-angle annular dark-field (HAADF) imaging and EDS mapping of the FIB section show that the refractory nugget is flanked by spinel $\left(\mathrm{MgAl}_{1.99} \mathrm{O}_{4}\right)$ on one side and melilite $\left(\mathrm{Ca}_{1.92} \mathrm{Al}_{1.68} \mathrm{Mg}_{0.18} \mathrm{Si}_{1.19} \mathrm{O}_{7}\right)$ on the other (Fig. 2). EDS reveals that Bright2 is compositionally heterogeneous (Fig. 2) with spatial correlations 
among $\mathrm{Fe}$, Ni and Pt, and also among Os, Ir, and Ru (Fig. 2). Mo is observed alongside $\mathrm{O}$ and Fe (Fig. 2). SAED patterns show that the refractory nugget is crystalline, and those acquired from the metallic regions rich in Os, Ir and Ru index to rutheniridosmine, aOs-Ir-Ru alloy. Thus, the EDS and SAED data show that Bright2 is composed of metallic alloys and oxides.

Previous descriptions of RMNs describe them as sub-micron- to micron-sized inclusions composed of singlephase alloys [1, 6-7]. Some RMNs contain cores enriched in $\mathrm{Os}, \mathrm{Ir}, \mathrm{Ru}$ and $\mathrm{Rh}$, and boundaries enriched in $\mathrm{Pt}$ [7], while others are composed of metallic cores surrounded by Ni-rich and Ni-poor Fe sulfides [8]. In comparison, our observations show that the size of Bright 2 is similar to previous descriptions of RMNs. While the presence of siderophiles such as Os, Ir, Ru and Pt is consistent with earlier measurements [1,6-7], the polyphasic nature of Bright 2 deviates from those descriptions of RMNs as being single-phase alloys. Further, Bright 2 is neither surrounded by Fe-sulfides nor a Pt-enriched rim. Earlier equilibrium thermodynamic calculations by [8] indicate that Os, Ir, Ru and Pt condense at $1917 \mathrm{~K}, 1639 \mathrm{~K}, 1613 \mathrm{~K}$, and $1415 \mathrm{~K}$ respectively. The same calculations also suggest that once a refractory metal condenses, it is thermodynamically favorable for the metal to incorporate other solutes, e.g., $\mathrm{W}, \mathrm{Fe}$ and $\mathrm{Ni}$, in levels proportional to their partial pressures in the solar nebula gas. Such alloying occurs at temperatures above those at which the host melilite $(1529 \mathrm{~K},[2])$ and adjacent spinel $(1397 \mathrm{~K}$, [2]) are predicted to condense. That Bright 2 occurs in a FTA CAI, itself a product of condensation, and contains $\mathrm{Os,} \mathrm{Ir}, \mathrm{Ru}$, and Pt in addition to $\mathrm{Fe}$ and $\mathrm{Ni}$ is consistent with the incorporation of solutes at high temperature from the nebular gas. The polyphasic nature of Bright2 was likely the result of such high-temperature alloying followed by localized oxidation in the solar nebula. We hypothesize that Bright 2 could represent an early nucleation seed for the condensation of the surrounding melilite and spinel. Additional work on this and other ultra-refractory grains will help test the above hypotheses.

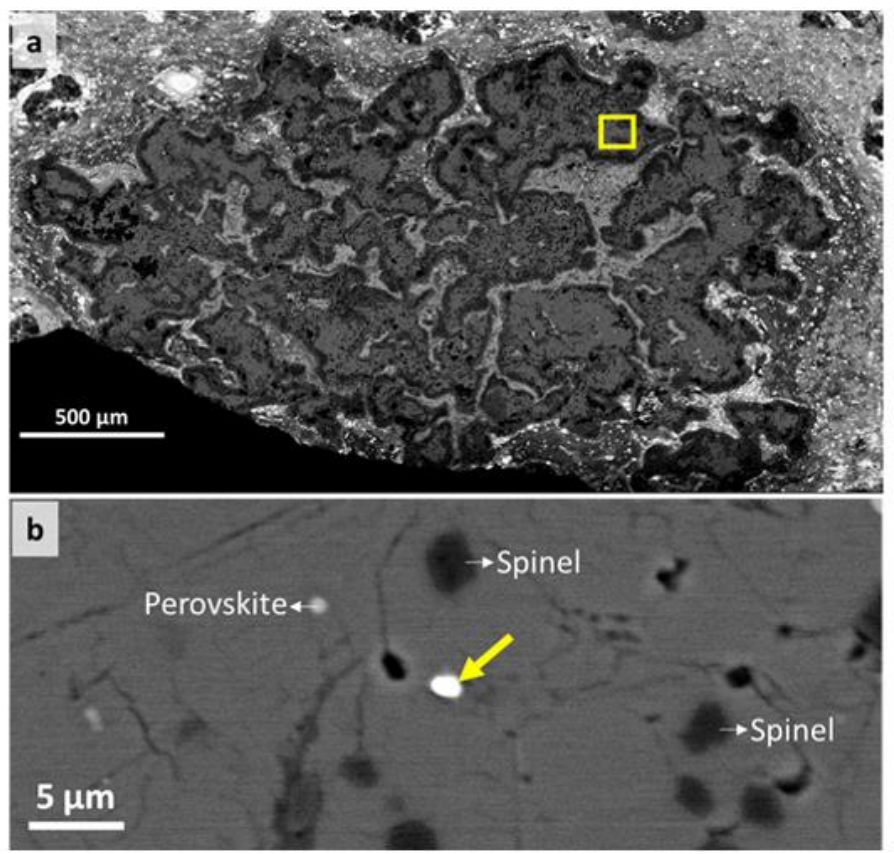

Figure

1. (a) BSE image of FTA 'Cloud' and the surrounding matrix. The yellow box indicated the region in which Bright 2 occurs. (b) BSE image of the local part of Cloud containing Bright2. Spinel and perovskite grains, and Bright 2 are surrounded by melilite. 

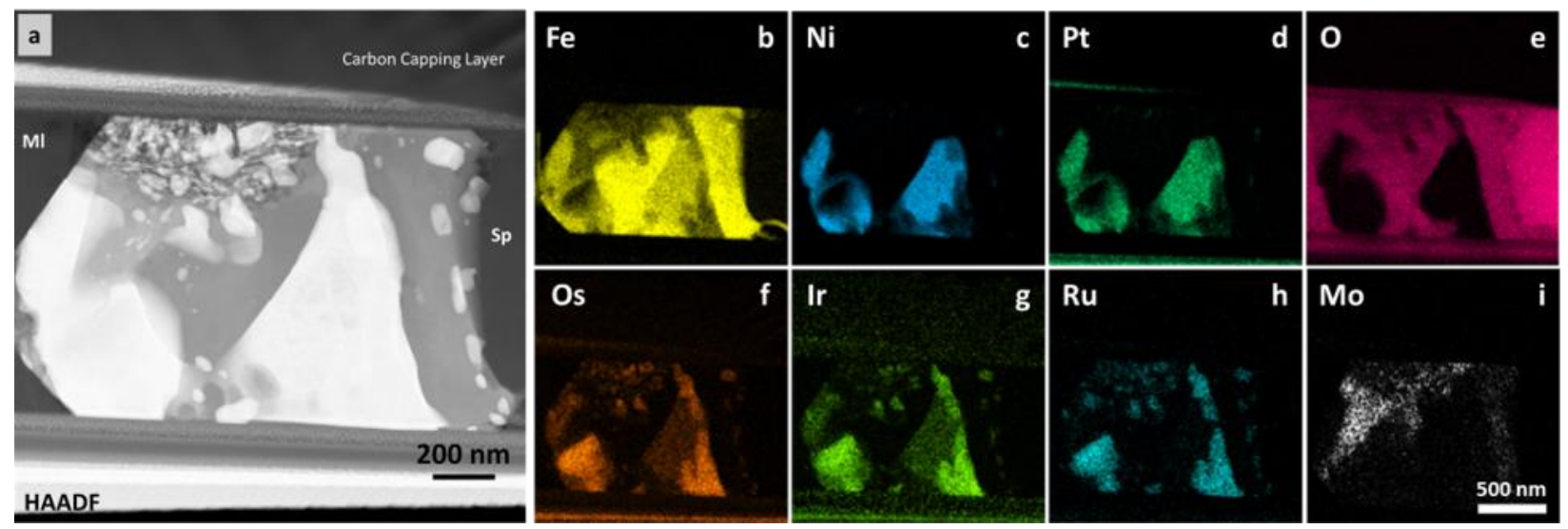

Figure 2. (a) HAADF image of Bright2. $\mathrm{Ml}=$ Melilite $\mathrm{Sp}=$ Spinel. (b) to (i) False-color EDS maps of Bright2.

\section{References}

[1] MacPherson G. J. in "Treatise on Geochemistry. Vol I: Meteorites, Comets and Planets", ed. Davis A.M., (Elsevier, Waltham) p. 201.

[2] Lodders K., The Astrophysical Journal 591 (2003), p. 1220.

[3] Ebel D.S. in "Meteorites and the Early Solar System II", ed. Lauretta D.S. and McSween Jr. H.Y., (University of Arizona Press, Tucson) p. 253.

[4] Amelin Y., Science 297 (2002), p. 1678.

[5] Connelly J.N. et.al., Science 338 (2012), p. 651.

[6] Wark D. A. and Lovering J. F., Lunar \& Planetary sciences Conference (1976), Abstract \#1317.

[7] El Goresy A. et.al., Lunar \& Planetary sciences Conference (1978), Abstract \#1100.

[8] Palme H. and Wlotzka F., Earth and Planetary Science Letters 33 (1976), p. 45.

[9] Berg T. et.al., The Astrophysical Journal 702 (2009), p. 172.

[10] Liffman K. et.al., Icarus 221 (2012), p. 89.

[11] Zega T. J., et. al., Meteoritics \& Planetary Science 42 (2007), p. 1373. 